\title{
Clinical Analysis of Treating Patients with Lumbar Interver- tebral Disc Using Chinese Medical Massage Therapy Com- bined with Needle Warming Moxibustion Therapy
}

\author{
C. LIANG*, T. JIANG, SHAOPING CHEN, Y. ZHANG, YIKUN CHEN, M. ZHANG, YANGLI OU AND MINGHUI LIN \\ Department of Acupuncture and Moxibustion, Haikou hospital of traditional Chinese Medicine, Haikou 570216, China
}

Liang et al.: Chinese Medical Massage Therapy Combined with Needle Warming Moxibustion Therapy

To observe and analyze clinical effect of combination therapy of Chinese medical massage and needle warming moxibustion in treating lumbar intervertebral disc. 148 patients with lumbar intervertebral disc who had been treated in our hospital from August 2015 to August 2016 were selected as research objects. These patients were randomly divided into control group which was given with needle warming moxibustion and research group which was given with combination therapy of Chinese medical massage and needle warming moxibustion, each containing 74 patients. The overall effective rates and Japanese orthopaedic association scoring improvement indexes of groups as well as the symptom grade and visual analogue scale before and after treatment of both groups were observed and compared. After treatment, the overall effective rate of research group was significantly higher than of control group, $\mathbf{p}<0.05$. In terms of Japanese orthopaedic association scoring improvement indexes and visual analogue scale scoring, research group had significant advantage over control group. Before treatment, the symptom scores of both groups were of no significant difference, $\mathbf{p}>\mathbf{0 . 0 5}$. After treatment, the improvement degree of control group was significantly lower than that of research group, with statistical significance, $p<0.05$. The combination therapy of Chinese medical massage and needle warming moxibustion can achieve ideal therapeutic effect in treating lumbar intervertebral disc, significantly relieve clinical symptoms and improve living quality of patients. Therefore, such combination therapy is worthy of being promoted in clinical application.

Key words: Chinese medical massage, needle warming moxibustion, lumbar intervertebral disc, clinical effect

Lumbar intervertebral disc is a major cause for low back and leg pain. Domestic and foreign scholars have conducted number of researches and pointed out that there are many causes for lumbar intervertebral disc such as genetic factor, immune and physiological structure, injury or degeneration of lumbar intervertebral disc, gestation. Lumbar intervertebral disc brings severe impact to patient's normal work and living quality. According to relevant research data, there are 1.5 million people suffering such disease per year in China, which is still on a rising trend ${ }^{[1]}$.

Lumbar intervertebral disc (fig. 1) is more likely to occur among elderly people, followed by middle aged people. Caused by many factors such as degeneration of intervertebral disc (fig. 2), annular disruption (fig. 3 ), herniation of the nucleus pulposus and oppression of cauda equina nerve and nerve root (fig. 4), lumbar intervertebral disc can make patients difficult to stand up and cause sharp pain which is hardly to bear in serious condition after attack ${ }^{[2]}$.

Chinese medicine therapy can achieve good therapeutic effect in treating lumbar intervertebral disc, which has been accepted and concerned by massive amount of patients. On this basis, this research observed and analyzed the therapeutic effect of combination therapy of Chinese medical massage and needle warming moxibustion.

*Address for correspondence

E-mail: Ic8418@163.com 


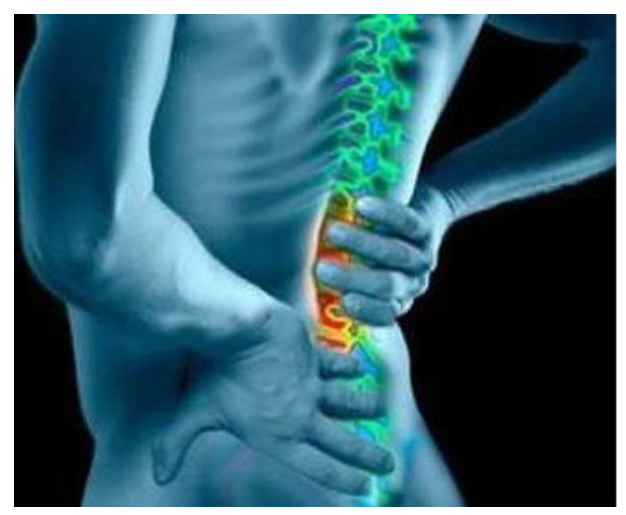

Fig. 1: Lumbar intervertebral disc

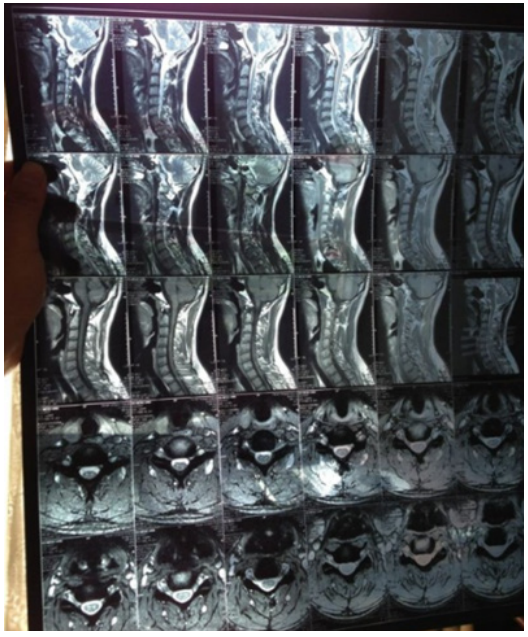

Fig. 2: Degeneration of intervertebral disc

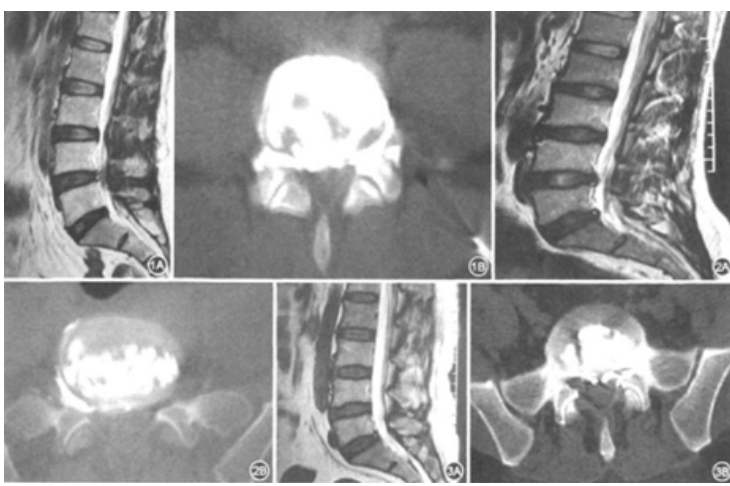

Fig. 3: Annular disruption

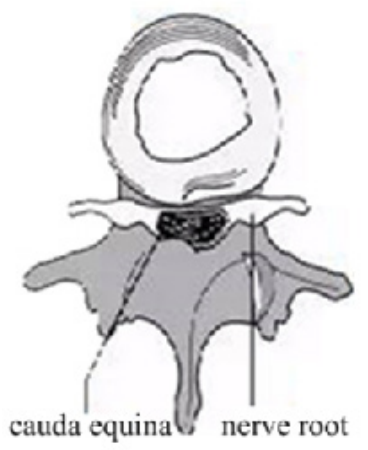

\section{MATERIALS AND METHODS}

\section{General data}

148 patients with lumbar intervertebral disc who had been treated in our hospital from August 2015 to August 2016 were selected as research objects. All patients were applied with X-ray, computed tomography (CT), magnetic resonance imaging (MRI) examination and confirmed to meet some criteria of lumbar intervertebral disc, with major symptoms including sciatica (fig. 5), low back and leg pain. Meanwhile, those with heart, liver and kidney dysfunctions and hypertension were excluded. All patients and relatives enjoyed the right to know and signed informed consent forms. All patients were randomly divided into control group and research group, each containing 74 patients. Research group contained 28 female patients and 46 male patients, with age distribution from 32 to 76 , averaging at $45.8 \pm 6.2$ y old; control groups contained 30 female patients and 44 male patients, with age ranging from 30 to 75 , averaging at $43.5 \pm 5.6$ y old. Through comparing general data of both groups, there existed statistical significance, $\mathrm{p}>0.05$.

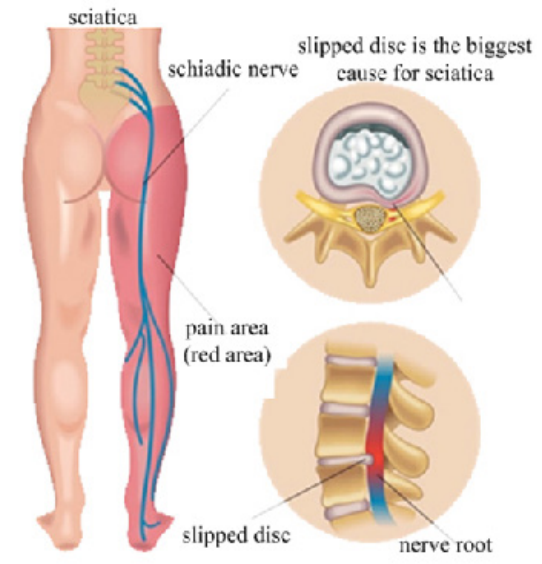

Fig. 5: Sciatica

\section{Methods}

Control group was applied with needle warming moxibustion therapy. Patients in control group were kept in lateral position and given with needle warming moxibustion therapy at corresponding acupoints (huantiao point, shenshu point, yaojia point, weizhong point, sanyinjiao point and yanglingquan point) according to actual condition of each patient ${ }^{[3]}$. Needle therapy was given once for $1 / 2 \mathrm{~h}$ (let patient feel warm), once a time and for 10 days, for the course of treatment ${ }^{[4]}$.

Fig. 4: Cauda equina 
Research group was given with combination therapy of Chinese medical massage and needle warming moxibustion. Patients in research group were kept in prone position and given with massage at waist, leg and hip area ${ }^{[5]}$, once for $3 \mathrm{~d}$ and $10 \mathrm{~d}$ for a course of treatment.

\section{Observation indexes}

Observe and record (lower back and leg pain) Japanese orthopaedic association (JOA) scores of both groups, wherein higher score means higher degree of pain; record symptom scores and visual analogue scale (VAS) scores before and after treatment of both group; observe overall effective rates of both groups, including recovery, significantly effective, effective, non-effective. The criteria of recovery includes JOA score lower than 4 points, disappearance of all clinical symptoms, straight leg raising to 90 degree, basically disappearance of dyskinesia and sensory disturbance, capability of living normal life and working normally ${ }^{[6]}$. The criteria of significant effectiveness includes JOA score ranging from 4-8, straight leg raising to around 70 degree, basically normal motion and sensory function, significantly relieved clinical symptoms, recovered working and living quality ${ }^{[7]}$; the criteria of effectiveness includes JOA score ranging 9-12 points and improved indexes; the criteria of non-effectiveness includes JOA score over 12 points, unimproved symptoms and indexes.

\section{Statistical method}

In this research, SPSS 21.0 statistical software was adopted to process and analyze data, wherein enumeration data was expressed by (n, \%) and tested by chi square, while measurement data was expressed by $( \pm \mathrm{s})$ and tested by $\mathrm{t}, \mathrm{p}<0.05$, with statistical significance.

\section{RESULTS AND DISCUSSION}

As shown in Table 1, the overall treatment efficiency of research group is significantly higher than that of control group, $\mathrm{p}<0.05$.

As shown in Table 2 the symptom scores of research group after treatment are significantly higher than before treatment and the symptom scores of research group are significantly higher than that of control group, $p<0.05$.

As shown in Table 3, the JOA score improvement indexes of both groups are tested by independent sample $t$, with statistical significance, $p<0.05$. The improvement degree of JOA scores of research group is significantly higher than that of control group.

As shown in Table 4, before treatment there is no significant difference of VAS scores between both groups, without statistical significance, $\mathrm{p}>0.05$; after treatment, the VAS score of research group is significantly reduced, which is superior than that of control group, $\mathrm{p}<0.05$.

TABLE 1: COMPARISON OF OVERALL TREATMENT EFFICIENCIES OF BOTH GROUPS [N, (\%)]

\begin{tabular}{lcccccc}
\hline Group & $\begin{array}{c}\text { Number of } \\
\text { patients }\end{array}$ & Recovery & $\begin{array}{c}\text { Significantly } \\
\text { effective }\end{array}$ & Effective & Non-effective & $\begin{array}{c}\text { Overall } \\
\text { effective rate }\end{array}$ \\
\hline Research group & 74 & $32(43.24)$ & $28(37.84)$ & $11(14.86)$ & $3(4.05)$ & $71(95.95)$ \\
Control group & 74 & $20(27.03)$ & $12(16.22)$ & $27(36.49)$ & $15(20.27)$ & $59(79.73)$ \\
$\mathrm{X}^{2}$ & & & & & 12.06 \\
$\mathrm{p}$ & & & & & $<0.05$ \\
\hline
\end{tabular}

TABLE 2: COMPARISON OF SYMPTOM SCORES OF BOTH GROUPS BEFORE AND AFTER TREATMENT ( $\overline{\mathrm{x}} \pm \mathbf{s})$

\begin{tabular}{lccc}
\hline Group & Number of patients & Before treatment & After treatment \\
\hline Research group & 74 & $10.72 \pm 3.78$ & $22.36 \pm 6.58$ \\
Control group & 74 & $10.56 \pm 4.13$ & $17.39 \pm 8.07$ \\
$\mathrm{t}$ & & 0.24 & 6.79 \\
$\mathrm{p}$ & & $>0.05$ & $<0.05$ \\
\hline
\end{tabular}


www.ijpsonline.com

TABLE 3: COMPARISON OF IMPROVEMENT CONDITIONS OF JOA SCORES OF BOTH GROUPS ( $\overline{\mathrm{x}} \pm \mathbf{s})$

\begin{tabular}{lcc}
\hline Group & Number of patients & JOA score improvement indexes (\%) \\
\hline Research group & 74 & $50.12 \pm 14.50$ \\
Control group & 74 & $38.06 \pm 16.23$ \\
$\mathrm{t}$ & & 12.39 \\
$\mathrm{p}$ & $<0.05$ \\
\hline
\end{tabular}

TABLE 4: COMPARISON OF VAS SCORES OF BOTH GROUPS ( $\bar{x} \pm \mathbf{s})$

\begin{tabular}{lccc}
\hline Group & Number of patients & Before treatment & After treatment \\
\hline Research group & 74 & $6.24 \pm 2.03$ & $1.56 \pm 2.83$ \\
Control group & 74 & $6.29 \pm 1.96$ & $3.79 \pm 3.31$ \\
$\mathrm{t}$ & & 0.11 & 8.72 \\
$\mathrm{p}$ & $>0.05$ & $<0.05$ \\
\hline
\end{tabular}

The major cause for lumbar intervertebral disc is the degenerative change of intervertebral disc. During the onset of such disease, the patient suffers intolerable pain, feels hard to sit and stand and experiences great impact on working and living quality. Western drug therapy can relieve the condition in certain degree but cannot affect a radical cure. Therefore, a correct and effective treatment scheme for lumbar intervertebral disc has become a key issue in current medical community.

In the perspective of traditional Chinese medicine, lumbar intervertebral disc belongs to a type of arthralgia, with typical symptoms of waist pain, lower limb radioactive numb and pain. The principle of treatment is to tonify qi, stimulate the menstrual flow and promote the circulation of blood. Acupuncture and moxibustion of traditional Chinese can make central pivot release substances to relieve pain as well as release opioid peptide to enhance immunity ${ }^{[8-10]}$. In addition, it can effectively improve perineural microcirculation, so as to prevent the conduction of nociceptive information and relieve myospasm. The principle of treating lumbar intervertebral disc by Chinese medical massage therapy is to reduce or eliminate the stimulation and compression of nucleus pulposus to nerve root, loosen the adhesion of nerve root, realize the reset or displacement of prolapsed nucleus pulposus and finally eliminate the sense of pain ${ }^{[11,12]}$. In addition, it can also conduct method of attack at vital point and massage to further improve therapeutic effect.

This research shows that the combination therapy of Chinese medical massage and needle warming moxibustion can achieve ideal therapeutic effect in treating lumbar intervertebral disc. Such combination therapy can significantly improve microcirculation at lesion area, not only relax the muscles and stimulate the blood circulation, but also relieve the pressured state of nerve root and achieve high overall treatment effective rate.

In conclusion, the combination therapy of Chinese medical massage and needle warming moxibustion can achieve more ideal therapeutic effect in treating lumbar intervertebral disc. On one hand, such combination therapy can significantly improve clinical symptoms and enhance patients living quality, on the other hand, it can help to eliminate inflammatory edema and thus effectively reduce sense of pain. Therefore, such combination method is worthy of being promoted in clinical application.

\section{Acknowledgements:}

2019, Research project of Hainan provincial health and family planning commission, (No. 19A200100), 2015 Hainan Natural Science Foundation Project (No. 20158371), 2015 Research project of Hainan provincial health and family planning commission (No. 15A200061).

\section{Conflicts of Interest:}

The authors declared no conflict of interest.

\section{REFERENCES}

1. Ma Q. Clinical analysis of treating 56 patients with lumbar intervertebral disc using combination therapy of Chinese medical massage and needle warming moxibustion. J Clin Med Lit 2015;11(4):642-4. 
2. Zhu FX, Yu ZF. Clinical efficacy of warm acupuncture combined with massage and traditional Chinese medicine nursing in the treatment of patients with lumbar disc herniation. Diabetes World 2019;16(12):27.

3. Li YD. Massage combined with warm acupuncture in the treatment of blood stasis type lumbar disc herniation. Guangming J Chin Med 2017;32(12):1772-3.

4. Wang LY, Yang J, Xiao YQ. Acupuncture with round-sharp needle guided by $\mathrm{X}$-ray combined with massage to correct lumbar physiological curvature in treatment of lumbar disc herniation. Int Med Health Guid News 2020;26(16):2359-61.

5. Wu ZX. Clinical effect of massage combined with Jiaji point warm acupuncture in the treatment of lumbar disc herniation. Chin Foreign Med Res 2017;15(4):114-6.

6. Lu F. Objective to analyze the effect of massage combined with warm acupuncture at Jiaji point on patients with lumbar disc herniation. China Health Care Nutr 2019;29(13):326-7.

7. Luo SX. Clinical observation on 35 cases of prolapse of lumbar intervertebral disc treated by massage combined with warm acupuncture and moxibustion. Shenzhen J Integr Tradit Chin West Med 2016;26(8):54-5.

8. Wang G, Li L, Zhi SB. The random parallel control study of massage, acupuncture combined Chinese medicine fumigation in the treatment of lumbar disc herniation. J Pract Tradit Chin Intern Med 2015;29(1):133-5.
9. Wang Z, Mou MW, Sun GR. Clinical curative effect of low temperature plasma radiofrequency nucleoplasty in the treatment of lumbar intervetebral disc herniation. China Med Her 2014;11(13):48-50.

10. Chen YG. Clinical observation on the treatment of lumbar disc herniation with cold dampness syndrome by external application of Wentong ointment on governor vessel combined with ginger moxibustion. J Clin Med Lit 2017;4(40):7772-3.

11. Zhao Y. Clinical study on acupuncture combined with massage in the treatment of lumbar disc herniation. Jilin J Tradit Chin Med 2011;31(4):344-5.

12. Zhang SL, Chen HS. 66 cases of lumbar disc herniation treated by warm acupuncture combined with acupoint injection. Fujian J Tradit Chin Med 2004;35(6):33-4.

This is an open access article distributed under the terms of the Creative Commons Attribution-NonCommercial-ShareAlike 3.0 License, which allows others to remix, tweak, and build upon the work non-commercially, as long as the author is credited and the new creations are licensed under the identical terms

This article was originally published in a special issue, "Trends in therapeutic Management of Various Clinical

Conditions II" Indian J Pharm Sci 2021:83(2)Spl issue;136-140 